\title{
Miedo a la madurez en adolescentes con anorexia nerviosa
}

\author{
Isabel Laporta Herrero ${ }^{1}$, Teresa Díez Martín², Patricia Latorre Forcén ${ }^{3}$, \\ Eric Vives Hidalgo ${ }^{3}$ y Víctor Navalón Monllor ${ }^{3}$ \\ ${ }^{1}$ Universidad Nacional de Educación a Distancia (España); \\ ${ }^{2}$ Hospital Universitario Lozano Blesa, Zaragoza (España); \\ ${ }^{3}$ Hospital Obispo Polanco, Teruel (España)
}

\begin{abstract}
El miedo a la madurez supone el deseo de regresar a la seguridad de la infancia y se considera uno de los constructos psicológicos que se ha mostrado clínicamente relevante en personas con anorexia nerviosa (AN). El estudio pretende determinar la proporción de pacientes con AN en nuestra muestra de estudio que presenta miedo a la madurez, y examinar si existen diferencias entre pacientes con AN restrictiva (AN-R) y AN purgativa (AN-P). La muestra se compone de 115 adolescentes diagnosticados de AN. Los pacientes cumplimentaron el Inventario de Trastornos de la Conducta Alimentaria (EDI-3) que incluye la variable psicológica miedo a la madurez. Los resultados indican que el $86.1 \%$ de la muestra presenta un rango clínico típico o elevado en la subescala miedo a la madurez y que no existen diferencias significativas entre los diagnósticos de AN-R y AN-P respecto al rasgo estudiado $(t=.160 ; p=.873)$. Este estudio pone de relieve la importancia de situar el foco en la práctica clínica cotidiana en el miedo a la madurez que presentan estos pacientes, tanto a nivel preventivo como en el tratamiento de la AN.
\end{abstract}

Palabras clave: Anorexia nerviosa, anorexia nerviosa restrictiva, anorexia nerviosa purgativa, adolescentes, miedo a la madurez.

Fear of maturity in adolescents with anorexia nervosa. Childhood and is considered one of the psychological constructs that has been clinically relevant for people with anorexia nervosa (AN). The study aims to determine the proportion of patients with AN in our study sample that presents fear of maturity, and examine if there are differences between patients with restrictive AN (AN-R) and purgative AN (AN-P). The sample consists of 115 adolescents diagnosed with AN. The patients completed the Inventory of Eating Disorders (EDI-3) that includes the psychological variable fear of maturity. The results indicate that $86.1 \%$ of the sample presents a typical or high clinical range in the fear of maturity subscale and there are no significant differences between the diagnoses of AN-R and AN-P $(t=.160$; $p=.873$ ). This study highlights the importance of focusing in daily clinical practice on the fear of maturity of these patients, both at a preventive level and in the treatment of AN.

Keywords: Anorexia nervosa, restrictive anorexia nervosa, purging anorexia nervosa, adolescents, fear of maturity.

Correspondencia: Isabel Laporta Herrero. Universidad Nacional de Educación a Distancia. C/ Tudela ${ }^{\circ} 11,2^{\circ}$. C.P.: 31003. Pamplona (España). E-mail: isabelaporta@ hotmail.com 
Los trastornos de la conducta alimentaria (TCA) son trastornos mentales caracterizados por alteraciones en el comportamiento alimentario, acompañados normalmente por intensa preocupación con el peso o la imagen corporal (APA, 2002) y generalmente tienen su inicio en la adolescencia (Rohde, Stice, y Marti, 2015). Entre las categorías diagnósticas de TCA (APA, 2002) se encuentra la anorexia nerviosa (AN) que se determina por una distorsión de la imagen corporal, rechazo a mantener un peso igual o por encima del valor mínimo normal considerando su edad y talla, y miedo intenso a ganar peso, lo que conduce a adoptar estrategias inadecuadas para prevenir su aumento como la realización de ejercicio físico excesivo y la restricción alimentaria, denominándose en este caso anorexia nerviosa restrictiva (AN-R). Si se asocia también a atracones de comida y/o conductas purgativas como la provocación del vómito o uso excesivo de laxantes, diuréticos o enemas, se denomina anorexia nerviosa purgativa (AN-P).

El miedo a la madurez supone el deseo de regresar a la seguridad de la infancia. Éste se considera uno de los constructos psicológicos que se ha mostrado clínicamente relevante en personas con AN (Behar-Astudillo y Arancibia-Meza, 2013) y un aspecto central en el mantenimiento de los TCA en el subgrupo de pacientes adolescentes en los que la pérdida de peso y la dieta juegan un papel funcional al proporcionar un medio de regresar a su apariencia y estado hormonal prepuberal, lo que les permite evadirse de la confusión, los conflictos y las expectativas de desarrollo asociadas a la adultez (Garner, 2004). De hecho, aparece como una subescala principal del Inventario de Trastornos de la Conducta Alimentaria (EDI) de Garner, Olmstead, y Polivy (1983) permaneciendo hasta la última revisión del instrumento (EDI-3) de Garner (2004). Según Bruch (1980) los pacientes con AN se sienten incapaces de enfrentarse a las contingencias biopsicosociales de la adolescencia, y los cambios propios del desarrollo como el incremento de la talla, silueta, peso y el inicio de la menstruación en las niñas, lo que representa un desafío peligroso para el cual no están preparados. Esta autora (1978) desde una posición psicodinámica identificó como un objetivo del tratamiento la necesidad de superar el "miedo a crecer y a madurar", afirmando que "muchos (pacientes) refiriendo que desean ser personas independientes, se mostrarán rápidamente de acuerdo y aceptarán como propio este objetivo, cuando en realidad todo su comportamiento refleja que ellos temen a la adultez y han tomado la nefasta resolución de no crecer" (p. 139). Por otro lado, Crisp (1967) desde una posición biológica relaciona el temor por la imagen corporal que desarrollan los pacientes con AN con un estado de inmadurez psicosexual, y el hacer dieta y el miedo al incremento de peso como una solución de regresión al estado prepuberal y un rechazo activo del rol sexual adulto. Por tanto, la restricción alimentaria que realizan los pacientes con AN sería el mecanismo utilizado para retornar a la apariencia, estado hormonal y experiencia prepubescente (Crisp, 1980). También señala que los cambios de la adolescencia pueden representar una amenaza para los otros miembros de la familia, lo 
cual ayudaría a mantener la patología alimentaria del hijo adolescente. Además, se ha asociado el miedo a la madurez con una personalidad insegura, incompetente en las relaciones, con sentimientos de ineficacia personal, desconfianza interpersonal y dificultades a nivel relacional (Grasso, Nazzaro, Vona, Capacchione, y Loriedo, 2012).

En cuanto a los estudios que comparan el miedo a la madurez en pacientes con AN-R y AN-P, Behar, Arriagada, y Casanova (2005) constataron que el tipo restrictivo de AN mostró puntajes significativamente mayores en la subescala miedo a la madurez del EDI en comparación con pacientes con otros trastornos mentales y estudiantes sin patología alimentaria ni afectiva. Woodside, Carter, y Blackmore (2004) por el contrario hallaron que el grupo de AN-P en contraste con AN-R exhibía un mayor miedo a la madurez y una mayor tasa de abandono en un programa de tratamiento hospitalario especializado.

Además este rasgo se ha asociado con un inicio precoz de la $\mathrm{AN}$, especialmente si se trata del subtipo restrictivo de la AN (Behar, 2010), a una escasa disposición al proceso terapéutico y a un mayor abandono del tratamiento de los pacientes con TCA (Fassino, Pierò, Tomba, y Abbate-Daga, 2009), proponiéndose este constructo como foco central de la psicoterapia con el objetivo de reducir este predictor de la pobre adherencia al tratamiento (Zeek, Hartmann, Buchholz, y Herzog, 2005).

Por ello, dada la relevancia que parece tener este constructo psicológico en el tratamiento de la AN y la contradicción de los resultados en la bibliografía actual, los objetivos del presente estudio son los siguientes: 1) determinar la proporción de pacientes con AN de nuestra muestra de estudio que presentan miedo a la madurez, y 2) examinar si existen diferencias entre pacientes con AN-R y AN-P en cuanto al miedo a la madurez.

\section{MÉTODO}

\section{Participantes}

La muestra se compone de 115 pacientes diagnosticados de AN según el DSM-IV-TR (APA, 2002). El 73.9\% presentan AN-R y 26.1\% AN-P. El $88.7 \%$ son mujeres y el $11.3 \%$ hombres, con edades comprendidas entre los 13 y 17 años $(M=14.76$; $D T=1.355$ ). Todos los pacientes acuden a una unidad específica infanto-juvenil de TCA del Sistema Nacional de Salud. El IMC medio de los pacientes es de 17.03 ( $D T=1.39)$.

En cuanto al lugar de residencia, el $34.8 \%$ viven en medios rurales y el $65.2 \%$ en medios urbanos. Todos son estudiantes, el $58.3 \%$ de la muestra acude a colegio público frente al $41.7 \%$ que asiste a colegio concertado-privado. Ver tabla 1. 
Tabla 1. Frecuencias y porcentajes de las variables sociodemográficas de la muestra de estudio $(n=115)$

\begin{tabular}{ccc}
\hline Variable & Frecuencia & Porcentaje \\
\hline Sexo & & \\
\hline -Hombre & 13 & $11.3 \%$ \\
\hline -Mujer & 102 & $88.7 \%$ \\
\hline Diagnóstico & & \\
\hline -Anorexia nerviosa restrictiva & 85 & $73.9 \%$ \\
\hline -Anorexia nerviosa purgativa & 30 & $26.1 \%$ \\
\hline Población & & \\
\hline -Medio rural & 40 & $34.8 \%$ \\
\hline -Medio urbano & 75 & $65.2 \%$ \\
\hline Centro escolar & & \\
\hline -Público & 67 & $58.3 \%$ \\
\hline -Concertado/privado & 48 & $41.7 \%$ \\
\hline
\end{tabular}

\section{Instrumentos}

Inventario de Trastornos de la Conducta Alimentaria (EDI-3. Eating Disorders Inventory-3) de Garner (2004) adaptado en población española por Elousa, López-Jáuregui, y Sánchez-Sánchez (2010). Evalúa la presencia de TCA y permite además la exploración de características psicológicas en estos pacientes. Está compuesto por 91 ítems, organizados en 12 escalas principales: tres escalas específicas de los TCA (insatisfacción corporal, bulimia y obsesión por la delgadez) y nueve escalas psicológicas generales (baja autoestima, alienación personal, inseguridad interpersonal, desconfianza interpersonal, déficit interoceptivo, desajuste emocional, ascetismo, perfeccionismo y miedo a madurar) que son altamente relevantes, pero no específicas, de los TCA. También proporciona 6 índices: uno específico de los TCA (riesgo de TCA), y cinco índices de constructos psicológicos integradores (ineficacia, problemas interpersonales, problemas afectivos, exceso de control y desajuste psicológico general). Obtiene altos niveles de consistencia interna en todos los grupos diagnósticos (Elousa et al., 2010). Para nuestro estudio, únicamente tuvimos en cuenta las puntuaciones de la escala psicológica miedo a la madurez, que está compuesta por 8 ítems.

\section{Procedimiento}

Los resultados de las puntuaciones del cuestionario fueron recogidos de forma retrospectiva de las historias clínicas, previo permiso de las personas responsables de la unidad. Este cuestionario forma parte del protocolo inicial de evaluación psicométrica que se realiza a cada paciente que acude a la unidad de TCA. Este protocolo se aplica el primer día que acude un paciente nuevo a la unidad y pretende medir aspectos clínicos, de personalidad, capacidad intelectual y mecanismos adaptativos.

Por cuestiones metodológicas y de homogeneización, se establecieron los siguientes criterios de inclusión: 1) cumplir los criterios diagnósticos del DSM-IV-TR (APA, 2002) para los subgrupos de AN: AN-R y AN-P, 2) tener entre 13 y 17 años, 3) cumplimiento del cuestionario en su totalidad. Los resultados del cuestionario junto con 
la información pertinente al diagnóstico, sexo, edad, población y centro escolar, fueron recogidos de las historias clínicas asignando un código a cada paciente de forma que la confidencialidad de la información y su anonimato quedaran totalmente garantizados.

\section{Análisis de los datos}

Los datos obtenidos fueron tratados mediante el paquete estadístico para Windows SPSS (Statistical Package for the Social Sciences) versión 19. Con respecto a los análisis estadísticos utilizados, en función de la naturaleza de las variables, se realizaron estadísticos descriptivos y una prueba $t$ de Student para muestras independientes.

Se trata de un diseño cuantitativo, retrospectivo, descriptivo y transversal o estático puesto que nos interesa estudiar el fenómeno en un momento temporal concreto.

\section{RESULTADOS}

En primer lugar, realizamos un análisis descriptivo de los resultados de la subescala miedo a la madurez del cuestionario EDI-3 en función de los rangos clínicos que proporciona el instrumento (Garner, 2004). Los rangos clínicos permiten al profesional saber si las puntuaciones que presenta un paciente en particular se sitúan por encima de lo que es habitual en los pacientes con TCA (rango clínico elevado), son similares a los que presentan otros pacientes con TCA (rango clínico típico) o se sitúan por debajo de lo que es habitual en los pacientes con TCA (rango clínico bajo). En muestras clínicas, un rango clínico elevado supone puntuaciones directas entre 15 y 32 que corresponden a un percentil igual o superior a 67 (puntuación $T \geq 53$ ) un rango clínico típico puntuaciones directas entre 8 a 14 con un percentil entre 25 y 66 (puntuación $T=43-52$ ) y un rango clínico bajo puntuaciones directas entre 0 y 7 con un percentil igual o inferior a 24 (puntuación $T \leq 42$ ).

Los resultados indican que el $86.1 \%$ de la muestra de estudio presentan un rango clínico típico o elevado en la subescala miedo a la madurez (Tabla 2).

Tabla 2. Rangos clínicos de las puntuaciones de la subescala miedo a la madurez del EDI-3 en la muestra de estudio $(n=115)$

\begin{tabular}{lcc}
\hline & Frecuencia & Porcentaje \\
\hline Rango clínico elevado & 39 & 33.9 \\
\hline Rango clínico típico & 60 & 52.2 \\
\hline Rango clínico bajo & 16 & 13.9 \\
\hline
\end{tabular}

Si tenemos en cuenta los dos grupos diagnósticos, el $85.9 \%$ de los pacientes con AN-R presentan un rango típico o elevado, frente al $86.7 \%$ del grupo AN-P (Tabla 3). Si dividimos la muestra en función del sexo, el $69.3 \%$ de los hombres muestran un rango típico o elevado en miedo a la madurez, frente al $88.2 \%$ de las mujeres. 
Tabla 3. Rangos clínicos de las puntuaciones de la subescala miedo a la madurez del EDI-3 en función del grupo diagnóstico

\begin{tabular}{|c|c|c|c|}
\hline & & Frecuencia & Porcentaje \\
\hline \multicolumn{4}{|l|}{ AN Restrictiva } \\
\hline & Rango clínico elevado & 31 & $36.5 \%$ \\
\hline & Rango clínico típico & 42 & $49.4 \%$ \\
\hline & Rango clínico bajo & 12 & $14.1 \%$ \\
\hline \multicolumn{4}{|l|}{ AN Purgativa } \\
\hline & Rango clínico elevado & 8 & $26.7 \%$ \\
\hline & Rango clínico típico & 18 & $60.0 \%$ \\
\hline & Rango clínico bajo & 4 & $13.3 \%$ \\
\hline
\end{tabular}

En segundo lugar, llevamos a cabo una prueba $t$ de Student para muestras independientes con las puntuaciones de la subescala miedo a la madurez según los grupos AN-R y AN-P. Los resultados indican que no existen diferencias significativas entre ambos con $t=.160$ y $p=.873$. Tal y como se observa en la tabla 4, el grupo AN-R obtiene puntuaciones similares $(M=54.74 ; D T=25.775)$ al grupo AN-P $(M=53.87 ; D T=25.800)$.

Tabla 4. Estadísticos de las puntuaciones de la subescala miedo a la madurez del EDI-3

\begin{tabular}{lcccc}
\multicolumn{4}{c}{ según los pacientes con AN-R y AN-P } \\
\hline & $N$ & Media & $D T$ & $\begin{array}{c}\text { Error típico de } \\
\text { la media }\end{array}$ \\
\hline Anorexia Nerviosa restrictiva & 85 & 54.74 & 25.775 & 2.796 \\
\hline Anorexia Nerviosa purgativa & 30 & 53.87 & 25.800 & 4.710 \\
\hline
\end{tabular}

\section{DISCUSIÓN Y CONCLUSIONES}

El primer objetivo de este estudio era determinar la proporción de pacientes con AN que acudían a la unidad de TCA que presentaban miedo a la madurez. Los resultados indican que más de las tres cuartas partes de la muestra estudiada refiere un fuerte deseo por regresar a la seguridad de la infancia y evitar así las exigencias asociadas al desarrollo y a la madurez. Según Garner (2004) existe la creencia de que las obligaciones y las exigencias de ser adulto son demasiado grandes y de que la infancia es el periodo más feliz en la vida de una persona. Estos resultados ponen de manifiesto la incapacidad de aceptación de la madurez psicosexual y de la futura adultez que presentan los adolescentes que desarrollan AN, lo que se manifiesta en miedo a los cambios psicológicos, sociales y biológicos asociados con un peso de adulto. La relevancia que este estudio pone en esta variable va en la línea de revisiones realizadas sobre el constructo psicológico como el de Behar-Astudillo y Arancibia-Meza (2013).

El segundo objetivo pretendía examinar si existían diferencias entre pacientes con AN-R y AN-P respecto al miedo a la madurez. Los resultados demuestran que no hay discrepancias entre grupos diagnósticos, ambos exhiben puntuaciones típicas o elevadas en miedo a la madurez. Estos hallazgos son contrarios a los estudios de Behar et al. (2015) y Woodside et al. (2004) que determinaban diferencias entre los tipos restrictivo y 
purgativo de la AN. Este estudio aporta como novedoso el analizar el rasgo miedo a la madurez en una muestra de adolescentes con AN. Hasta la fecha esta variable se ha tratado de forma inespecífica en la literatura científica sobre el TCA, y este estudio señala la relevancia de la misma. Además, no hemos encontrado estudios que evalúen específicamente este rasgo en población adolescente, y es precisamente, en esta etapa evolutiva cuando la intervención es necesaria para evitar la falta de adherencia y/o el abandono al tratamiento, y conseguir así la recuperación ponderal y la reducción de la patología alimentaria.

Sin embargo, el presente estudio tiene algunas limitaciones. La muestra no ha sido seleccionada de manera aleatoria, no se trata de un estudio experimental y las relaciones entre las variables podrían estar afectadas por el efecto de variables no controladas. Asimismo, se han utilizado medidas de autoinforme por lo que queda a criterio de los participantes la sinceridad de sus respuestas, a lo que se añade la negación y la minimización de la sintomatología que es frecuente en la AN y que afecta a los resultados psicométricos (Vitousek, Daly, y Heiser, 1991). Por otra parte, hay que tomar con cautela los resultados del estudio ya que la variable principal se ha medido con una subescala del EDI-3 y únicamente implica 8 ítems para su evaluación. No obstante, desde nuestro conocimiento, no existe un cuestionario, inventario o escala que evalúe específicamente este rasgo psicológico que se muestra tan relevante en la patología de la AN. Creemos, por tanto, que un cuestionario específico del miedo a la madurez podría conducir nuevas líneas de investigación y aportar resultados interesantes de cara a la prevención y al tratamiento de los pacientes con AN.

Como conclusión, este estudio pone de relieve la importancia de situar el foco en la práctica clínica cotidiana en el miedo a la madurez que presentan estos pacientes, tanto en los programas de prevención como en el tratamiento de la AN. Respecto a este último, se ha demostrado como programas psicoeducativos y terapia familiar consiguen una mejoría significativa en el mismo (Behar-Astudillo y Arancibia-Meza, 2013). Futuras investigaciones en el campo podrían estudiar la comorbilidad de este constructo con otras variables significativas, como ya lo han hecho Fassino et al. (2006) con el ascetismo, Fujimori et al. (2011) con el control de impulsos, o Brytek-Matera y Schiltz (2009) con la ansiedad. Entre las implicaciones para la práctica clínica al estudio cabe señalar la necesidad de fomentar la autonomía y la independencia de estos pacientes, así como la aceptación de los cambios psicológicos, sociales y biológicos inherentes a la etapa evolutiva de la adolescencia. 


\section{REFERENCIAS}

American Psychiatric Association. (2002). DSM-IV-TR: Manual diagnóstico y estadístico de los trastornos mentales. Barcelona: Masson.

Behar, R. (2010). Trastornos de la conducta alimentaria: Clínica y epidemiología. En R. Behar y G. Figueroa (Eds.), Trastornos de la conducta alimentaria. Segunda edición (pp. 121-147). Santiago de Chile: Editorial Mediterráneo.

Behar, R., Arriagada, M.I., y Casanova, D. (2005). Eating and affective disorders: A comparative study. Revista Médica de Chile, 133, 1407-1414.

Behar-Astudillo, R., y Arancibia-Meza, M. (2013). Temor a la madurez en la anorexia nerviosa. Revista Mexicana de Trastornos Alimentarios, 4, 143-152.

Bruch, H. (1978). The golden cage. Cambridge MA: Harvard University Press.

Bruch, H. (1980). Preconditions for the development of anorexia nervosa. American Journal of Psychoanalysis, 40, 169-172.

Brytek-Matera, A., y Schiltz, L. (2009). A cross-cultural examination of the relationship between negative mood states, body image and eating disorders. Bulletin of Social Science and Medicine Grand Duche Luxembourg, 1, 11-25.

Crisp, A. (1967). The possible significance of some behavioral correlates of weight and carbohydrate intake. Journal of Psychosomatic Research, 11, 117-131.

Crisp, A. (1980). Anorexia nervosa: Let me be. London: Academic Press.

Elousa, P., López-Jáuregui, A., y Sánchez-Sánchez, F. (2010). Manual técnico con la adaptación al euskera del Eating Disorder Inventory-3. Madrid: TEA Ediciones.

Fassino, S., Pierò, A., Gramaglia, C., Daga, G.A., Gandione, M., Rovera, G.G., y Bartocci, G. (2006). Clinical, pspychological, and personality correlates of asceticism in anorexia nervosa: From saint anorexia to pathologic perfectionism. Transcultural Psychiatry, 43, 600-614.

Fassino, S., Pierò, A., Tomba, E., y Abbate-Daga, G. (2009). Factors associated with dropout from treatment for eating disorders: a comprehensive literature review. $B M C$ Pychiatry, 9, 67.

Fujimori, A., Wada, Y., Yamashita, T., Choi, H., Nishizawa, S., Yamamoto, H., y Fukui, K. (2011). Parental bonding in patients with eating disorders and self-in-jurious behavior. Psychiatry and Clinical Neurosciences, 65, 272-279.

Garner, D. (2004). Eating Disorder Inventory-3. Professional Manual. Lutz, FL: Psychological Assessment Resources, Inc.

Garner, D., Olmstead, M., y Polivy, J. (1983). Development and validation of a multidimensional eating disorder inventory for anorexia nervosa and bulimia. The International Journal of Eating Disorders, 2, 15-34.

Grasso, M., Nazzaro, F., Vona, L., Capacchione, G., y Loriedo, C. (2012). Maturity fears and weight phobia in eating disorders: Research of a relationship. Rivista di Psichiatria, 47, 319-326.

Rohde, P., Stice, E., y Marti, C.N. (2015). Development and predictive effects of eating disorder risk factors during adolescence: Implications for prevention efforts. International Journal of Eating Disorders, 48(2), 187-198. 
Vitousek, K.B., Daly, J., y Heiser, C. (1991). Reconstructing the internal world of the eatingdisordered individual: Overcoming denial and distortion in self-report. International Journal of Eating Disorders, 10(6), 647-666.

Woodside, D.B., Carter, J.C., y Blackmore, E. (2004). Predictors of premature termination of inpatient treatment for anorexia nervosa. American Journal of Psychiatry, 161, 2277 2281.

Zeeck, A., Hartmann, A., Buchholz, C., y Herzog, T. (2005). Drops outs from in-patient treatment of anorexia nervosa. Acta Psychiatrica Scandinavica, 111, 29-37.

Recibido: 5 de junio de 2018

Recepción Modificaciones: 16 de agosto de 2018

Aceptado: 3 de noviembre de 2018 